\title{
Le verre \\ ne se cache plus pour mûrir
}

David Bouttes ${ }^{(1)}$, Élodie Boller ${ }^{(2)}$, Emmanuelle Gouillart ${ }^{(3)}$

et Damien Vandembroucq ${ }^{(4)}$ (damienvdb@pmmh.espci.fr)

(1) Saint-Gobain CREE (Centre de Recherches et d’Études Européen), 550 rue Alfred Jauffret, 84300 Cavaillon

(2) European Synchrotron Radiation Facility (ESRF), 38043 Grenoble Cedex 9

(3) Surface du Verre et Interfaces (UMR 125 CNRS/Saint-Gobain), BP 135, 93303 Aubervilliers Cedex

(4) Physique et Mécanique des Milieux Hétérogènes (UMR 7636 CNRS/ESPCI/Univ. Paris 6 UPMC/Univ. Paris 7 Diderot),

10 rue Vauquelin, 75005 Paris

De nombreux verres d'oxyde

binaires ou ternaires présentent

un domaine de séparation

de phase. Nous exposons ici

des résultats obtenus à haute

température sur un verre

de borosilicate de baryum.

Par des expériences

de microtomographie $X$

synchrotron, nous avons suivi

in situ le régime de mûrissement

qui suit la séparation de phase.

Cette technique d'imagerie

3D nous a permis

de caractériser finement

la croissance des domaines et

l'évolution de leur géométrie.

Dans le régime de mûrissement

visqueux ici à l'œuvre, nous

avons en particulier pu mettre

en évidence un phénomène

original de fragmentation

qui limite la validité d'une

description auto-similaire

de la croissance des domaines.

Les termes en italiques sont explicités dans le

glossaire, p. 20 .

\section{Quand deux verres} préférent se séparer...

À l'état liquide, de nombreux verres présentent un domaine d'immiscibilité et sont donc sujets à une séparation de phase. Par décomposition spinodale (ou par nucléation), deux phases forment des domaines interconnectés (ou des gouttelettes isolées) [1] : on parle de verres " démixés ". Il s'ensuit un régime de croissance des domaines, piloté par la minimisation des interfaces et du coût énergétique associé : le mûrissement [2]. Même si pour observer ces phénomènes dans les verres silicatés, il est nécessaire de travailler à haute température (typiquement dans une gamme $500^{\circ} \mathrm{C}-1500^{\circ} \mathrm{C}$ ), la physique à l'œuvre est essentiellement la même que dans des systèmes plus classiques de la matière molle : émulsions, suspensions colloïdales, gels, polymères, etc.

La séparation de phase est en fait un sujet classique de la physique des verres d'oxyde [3]. Une de ses premières applications industrielles fut le développement des verres Pyrex. Dans ces verres de borosilicate, la nucléation de gouttelettes nanométriques (2-3 nm) [4] permet en effet d'obtenir une matrice de composition très proche de la silice pure. À moindre coût (grâce à une température d'élaboration plus faible que pour la silice), il est ainsi possible de fabriquer un verre de faible coefficient de dilatation, résistant aux chocs thermiques.

Du point de vue technologique, la maitrise du mûrissement (donc de la croissance des domaines) dans les verres démixés est en outre porteuse d'applications variées. La possibilité de figer la structure d'un verre à un stade bien choisi du mûrissement par une trempe thermique permet en effet d'envisager de texturer ces matériaux à petite échelle, en jouant sur un simple paramètre de contrôle macroscopique : la température. Grâce au mûrissement consécutif à la séparation de phase, il est ainsi possible de créer des verres composites à l'échelle nano- ou micrométrique selon les composés et les gammes de température utilisés. Une application historique de cette idée est la réalisation de verres nanoporeux comme le Vycor, pour lequel une des phases séparées est dissoute par attaque acide. Ces verres nanoporeux ont rapidement trouvé de multiples applications, depuis la catalyse du fait de leur grande surface spécifique jusqu'à l'optique pour des couches ou des fibres à gradient d'indice [5].

\section{... on peut tomographier leur croissance en trois dimensions...}

Profitant des progrès récents de la microtomographie X à l'ESRF, nous avons pu suivre in situ le mûrissement d'un verre démixé à haute température $[6,7,8]$. Le verre choisi, un borosilicate de baryum, se décompose en une phase enrichie en silice et une autre enrichie en baryum. La différence de densité entre ces deux phases garantit un excellent contraste d'absorption aux rayons $\mathrm{X}$ et facilite le travail de traitement d'image. La nature vitreuse du matériau permet de contrôler la dynamique de croissance en jouant sur la température : une plage de quelques dizaines de degrés permet de modifier la viscosité des phases de plusieurs ordres de grandeur. Une spécificité de ce type de verre démixé est le très grand contraste de viscosité entre les deux phases, de plus de trois ordres de grandeur 
>>

dans toutes nos expériences. On verra que ce contraste de viscosité a une influence cruciale sur la dynamique.

L'expérience (fig. 1a) consiste à placer un échantillon de verre entre la source de rayons $\mathrm{X}$ et un détecteur bidimensionnel, dans lequel les rayons $\mathrm{X}$ sont convertis en lumière visible par un scintillateur. Cette lumière est transmise à une caméra qui peut enregistrer une projection en quelques millisecondes. L'utilisation d'une platine de rotation permet alors de prendre des images 2D d'absorption $\mathrm{X}$ de l'échantillon à différents angles. À partir de la collection de ces radiographies à un instant donné, on reconstruit une image 3D de l'échantillon représentant son absorption, et donc une carte de sa composition chimique à l'échelle micrométrique. Les volumes imagés dans le cadre de l'expérience sont de l'ordre de $(700 \mu \mathrm{m})^{3}$ et la résolution spatiale (le voxel) de $(0,7 \mu \mathrm{m})^{3}$. Le rayonnement synchrotron est suffisamment intense pour que le temps d'acquisition d'une série de radiographies soit de l'ordre de quelques dizaines de secondes, ce qui permet de suivre in situ le mûrissement d'un échantillon de verre liquide placé dans un four spécialement conçu pour laisser passer les rayons $\mathrm{X}$ (fig. 1). Un traitement d'image permet enfin d'identifier les phases, et de mesurer différentes caractéristiques géométriques des domaines : volumes, surfaces, épaisseurs, connectivités, nombre de boucles, courbures des interfaces, etc.

L'imagerie 3D donne ainsi accès aux détails de la morphologie et à l'évolution dans le temps des domaines, dont la taille varie de quelques microns à quelques centaines de microns en quelques minutes. L'intérêt de la technique est particulièrement marqué lorsqu'à la séparation de phase se sont formés des domaines interconnectés (ce qui est typiquement le cas suite à une décomposition spinodale avec un rapport volumique des phases à l'équilibre de l'ordre de 0,5). On accède alors directement aux informations de connectivité, qui sont délicates à déterminer par d'autres méthodes (diffraction ou coupes stéréologiques). On peut également suivre l'évolution au cours du temps d'une interface ou d'un domaine isolé, et ainsi comprendre les mécanismes locaux de la dynamique.

\section{... et on les voit mûrir sans pour autant changer.}

À partir de ces expériences, nous avons pu tester et vérifier la validité de quelques modèles classiques de physique statistique. Dans de nombreux systèmes, la croissance se caractérise par une invariance d'échelle dynamique, c'est-à-dire qu'après normalisation de la géométrie par une longueur caractéristique $l(t)$ (taille typique des domaines, voir paragraphe suivant), la géométrie (adimensionnée) est statistiquement la même. À chaque instant, la géométrie est donc donnée par cette longueur $l(\mathrm{t})$ et par une configuration de référence. La croissance des domaines s'écrit alors sous la forme simple d'une loi de puissance : $l(\mathrm{t})=\mathrm{At}^{\alpha}$, où $\mathrm{A}$ et $\alpha$ dépendent des caractéristiques physiques du système et du mode de transport : diffusion, hydrodynamique... Cette loi d'échelle dynamique est extrêmement puissante puisqu'elle permet a priori de prédire efficacement l'évolution de la forme des domaines au cours du temps.
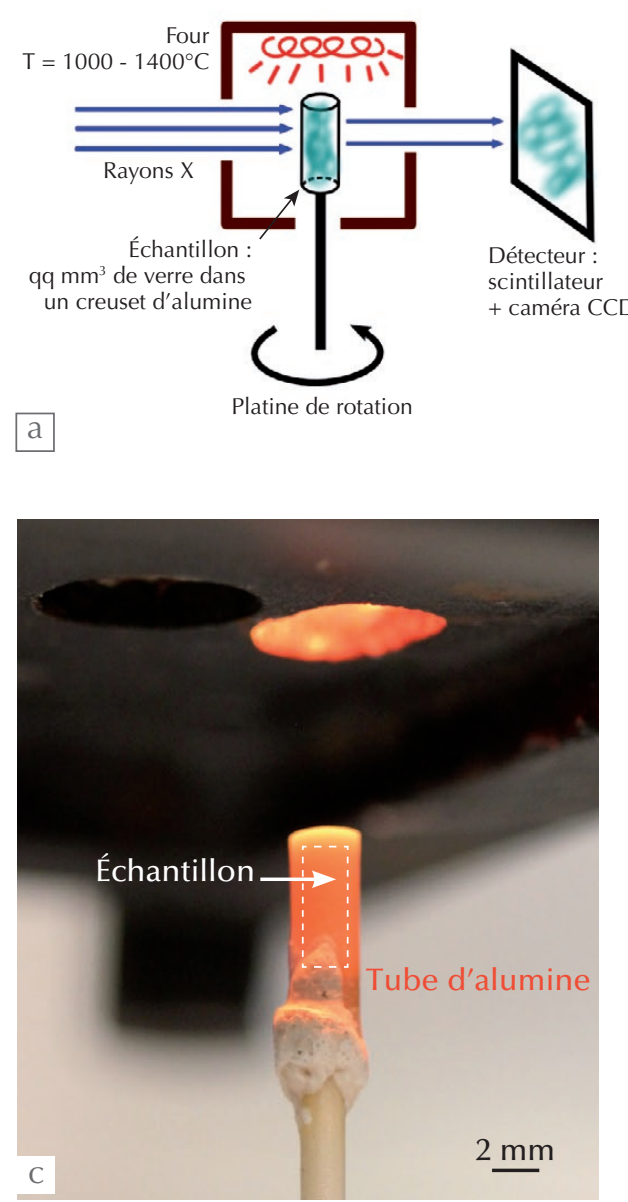

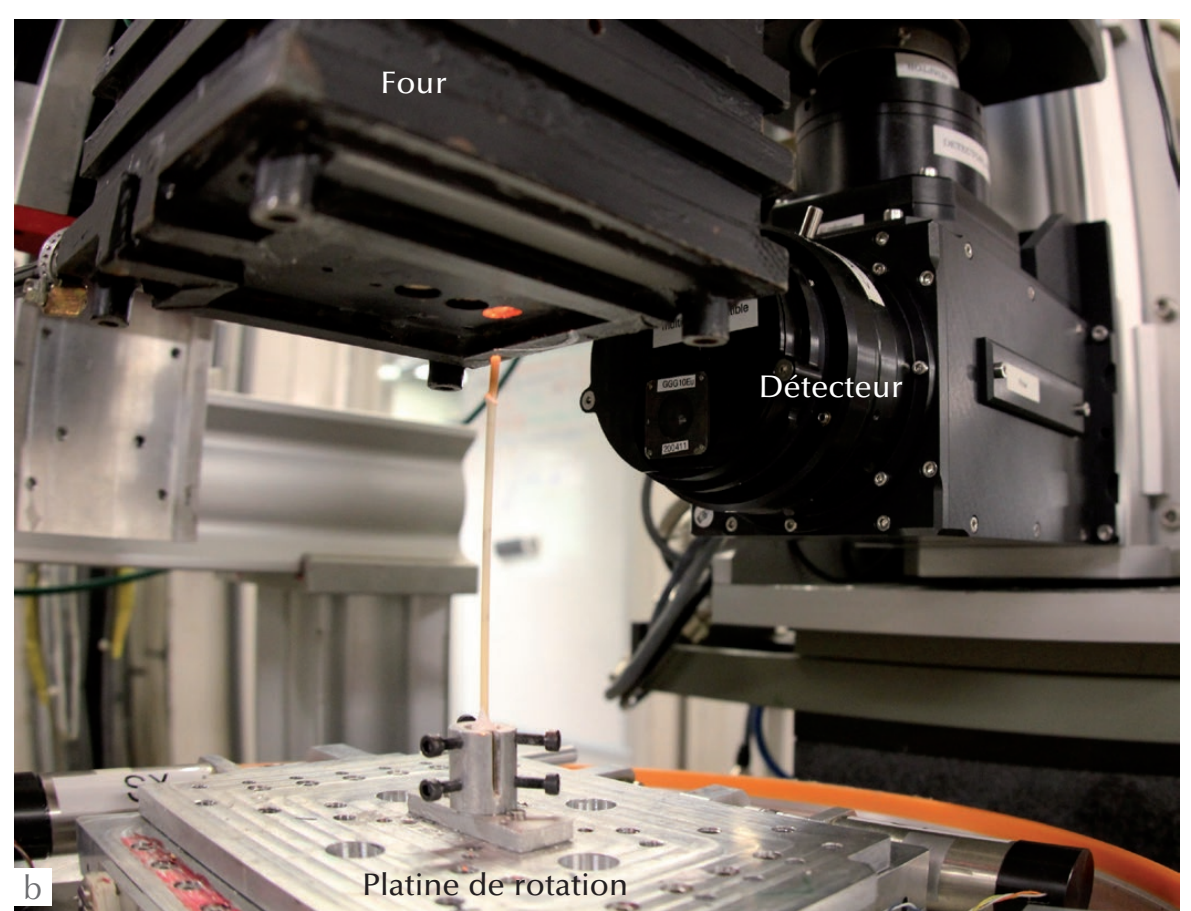

1. Dispositif expérimental sur la ligne ID19 à I'ESRF.

(a) Schéma de principe.

(b) Une photo de l'expérience, four relevé. L'échantillon, un cylindre millimétrique de verre, est placé dans un tube réfractaire d'alumine. La rotation de la platine permet d'enregistrer des séries de projections. Les expériences peuvent être réalisées in situ (four baissé, deux fenêtres laissent passer le faisceau de rayons X) ou ex situ, four levé. (c) Vue agrandie de l'échantillon dans son tube en alumine. 
Dans le système étudié, la mobilité est contrôlée par une hydrodynamique visqueuse : des domaines interconnectés grossissent par la suite de ruptures capillaires de ligaments. Chaque rupture entraîne la redistribution du fluide présent dans le ligament "rompu ", et vient faire grossir ses voisins (fig. 2). La dynamique du mûrissement dans ce régime a été proposée par Siggia en 1979 [9], et déjà confirmée par des expériences de diffraction. La loi d'échelle s'écrit comme un équilibre de force entre la tension de surface et les effets visqueux ; on obtient une croissance de la longueur caractéristique (le " diamètre " moyen des ligaments) linéaire en temps : $l(\mathrm{t}) \approx \gamma \mathrm{t} / \eta$, où $\gamma$ est la tension d'interface et $\eta$ la viscosité effective du système.

Les expériences de tomographie permettent de mettre en évidence la dynamique de mûrissement visqueux : en mesurant l'épaisseur moyenne des ligaments fluides (fig. 3), on observe bien que leur croissance est décrite par cette loi linéaire $l(t) \approx \gamma t / \eta$, avec un préfacteur de l'ordre de 1 . On retrouve ainsi la même microstructure (au sens statistique) à des échelles différentes. Les images 3D permettent en outre de confirmer le rôle des ruptures dans la croissance : dans ce système de domaines entremêlés, l'évolution de la topologie, c'est-à-dire du nombre de boucles des ligaments liquides, est indissociable de leur grossissement. Plus les ligaments sont épais, moins on compte de boucles (fig. 2). Une analyse quantitative des images nous a de plus permis de vérifier que l'évolution temporelle de la distribution des courbures locales, comme celle de la caractéristique d'Euler (qui caractérise la topologie des domaines), obéissaient bien à l'hypothèse d'invariance d'échelle dynamique [6].

\section{Mais l'un des deux verres se met à fragmenter...}

L'observation à temps long du mûrissement a pourtant mis en évidence un accroc de taille à cette description en loi d'échelle dynamique : la fragmentation de la phase la moins visqueuse. Grâce à l'imagerie 3D, on voit qu'initialement il n'y a qu'un seul domaine de chacune des phases, et que ces deux domaines s'entrecroisent. Le système est "bicontinu ", au sens où les phases percolent : on peut aller d'un bout à l'autre du système en suivant l'une ou l'autre phase. Au cours de la croissance, on
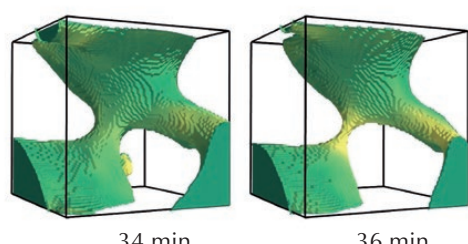

$36 \min$
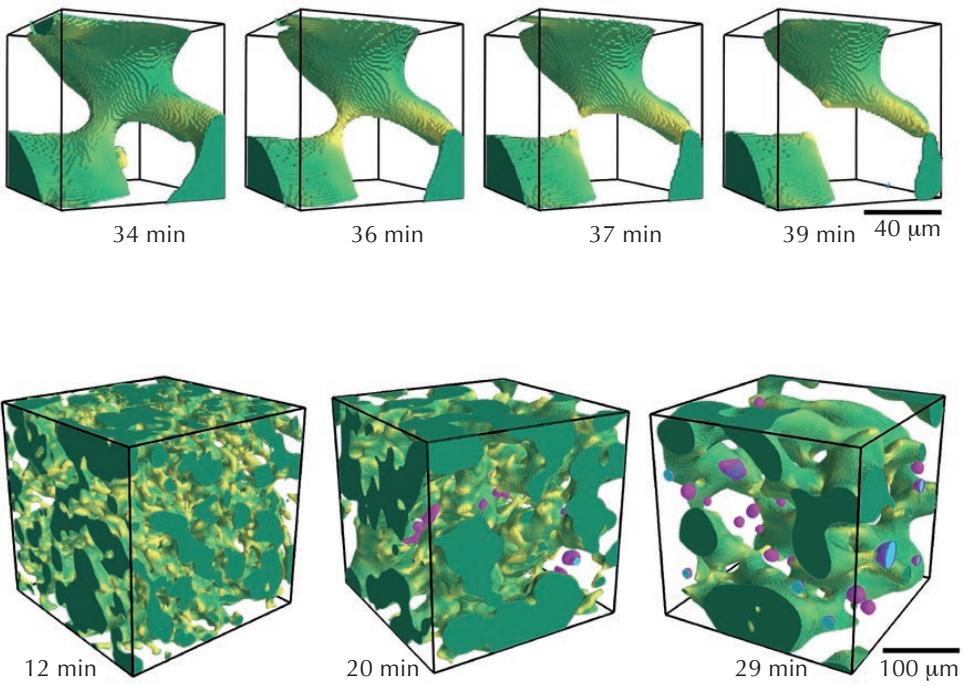

2. Tomographie $X$ du mûrissement d'un verre borosilicate de baryum à $1130^{\circ} \mathrm{C}$. Seule la phase minoritaire, riche en baryum et moins visqueuse, est ici représentée ; elle occupe environ $40 \%$ du volume. Le domaine percolant est représenté en nuances de vert, en fonction de la courbure locale : les zones de forte courbure sont plus claires; les domaines isolés sont en nuances de violet ; les intersections avec les limites de l'image sont en vert foncé et cyan, respectivement pour le domaine percolant et les domaines isolés. En haut, un événement local de pincement capillaire entraîne la réorganisation des domaines. En bas, une vision plus large permet d’apprécier l'augmentation de la taille caractéristique et, dans cette expérience, de la fragmentation progressive.

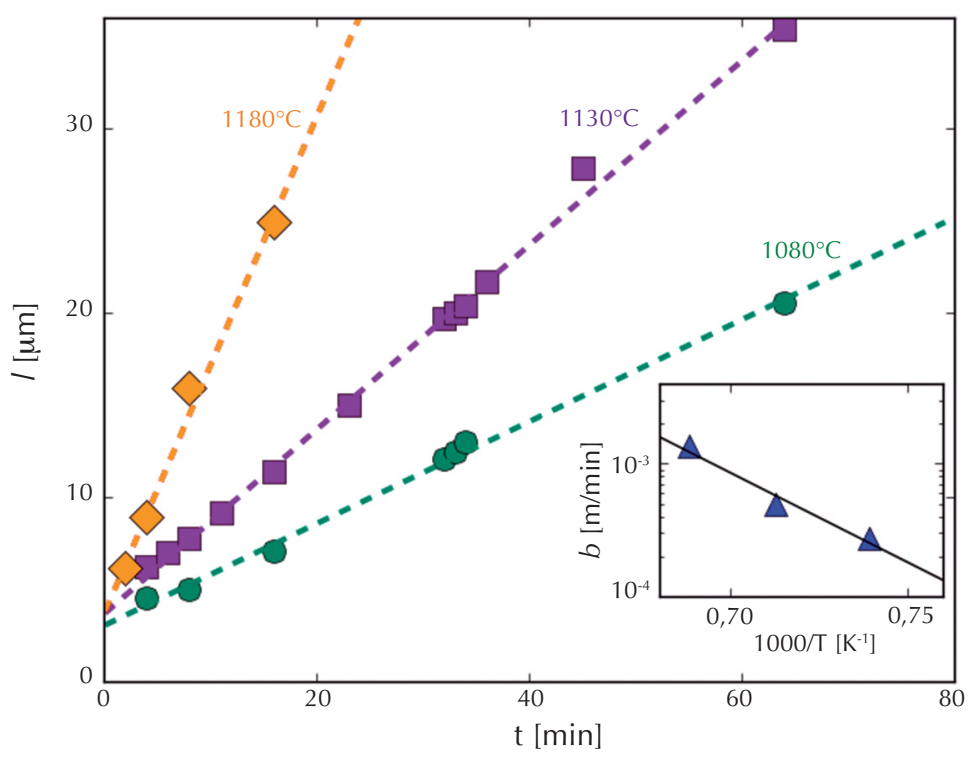

3. Évolution temporelle de la longueur caractéristique $I(T, t)$ lors du mûrissement d'un verre borosilicate de baryum à différentes températures $\mathrm{T}=1080^{\circ} \mathrm{C}, 1130^{\circ} \mathrm{C}$ et $1180^{\circ} \mathrm{C}$. Comme attendu dans le cadre d'un mûrissement visqueux, la croissance est linéaire avec le temps, avec une vitesse $b=\mathrm{d} /$ /dt d'autant plus rapide que la température est élevée. L'encart en bas à droite montre que l'évolution de cette vitesse $b$ avec la température obéit à un comportement de type Arrhenius, compatible avec la prédiction de Siggia : $I(T, t) \approx \gamma t / \eta(T)$ 


\section{Glossaire}

\section{Verres d'oxyde}

Lorsqu' on les refroidit suffisamment vite, certains liquides échappent facilement à la cristallisation et passent dans un état surfondu. Lorsqu'on continue à refroidir, la viscosité augmente si rapidement que les réarrangements moléculaires deviennent extrêmement lents. Le liquide finit par figer et former un verre. On parle de transition vitreuse. De nombreux composés peuvent former des verres. Les plus connus sont les verres d'oxyde, utilisés industriellement pour le vitrage, la verrerie, les produits d'isolation, les fibres optiques, etc. Dans la composition de ces verres on distingue classiquement des oxydes «formateurs de réseau » (tels $\mathrm{SiO}_{2}$ ou $\mathrm{B}_{2} \mathrm{O}_{3}$ ) qui vitrifient facilement, et des « modificateurs de réseau » (la plupart des oxydes d'alcalins ou d'alcalino-terreux), individuellement incapables de former des verres mais qui peuvent s'insérer au sein d'une structure vitreuse.

\section{Verre borosilicate}

Verre composé de silice $\mathrm{SiO}_{2}$, d'oxyde de bore $\mathrm{B}_{2} \mathrm{O}_{3}$ et généralement $d^{\prime}$ alcalins ou d'alcalino-terreux (sodium ou calcium par exemple, baryum dans notre cas). Les borosilicates sont couramment utilisés dans la verrerie en raison de leur bonne résistance chimique. Le verre à vitre ne contient pas d'oxyde de bore, mais de la silice associée à du sodium et du calcium.

\section{Séparation de phase}

Suivant les conditions de température et de pression, un mélange de composés $A$ et $B$ peut être thermodynamiquement stable et former une seule phase, ou être instable et tendre à se décomposer en deux phases stables $A^{\prime}$ et $B^{\prime}$ (respectivement riches en $A$ et en $B$ ). Lorsqu'un mélange à l'équilibre est " trempé » brutalement dans une région instable de l'espace des phases, il commence à démixer. En fonction des fractions volumiques de $A$ et $B$, cette séparation de phases peut se faire par nucléation de gouttelettes de $A^{\prime}$ (ou de $B^{\prime}$ ) au sein du mélange (fig. E1 gauche), ou en I'absence de barrière d'énergie, par décomposition spinodale (fig. E1 droite). Dans ce second cas, toute fluctuation de composition du mélange est alors instable, des domaines dont les compositions respectives se rapprochent graduellement des valeurs d'équilibre $A^{\prime}$ et $B^{\prime}$ se forment spontanément [10].

Nucléation
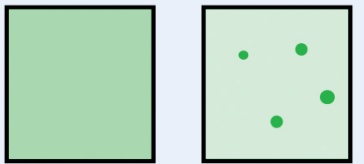

E1. Séparation de phase
Décomposition spinodale

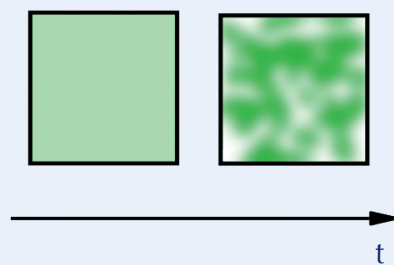

\section{Mûrissement}

Immédiatement après les premiers stades de la séparation de phase, le système est encore très loin de son état d'énergie minimale. Les domaines formés par nucléation ou décomposition spinodale sont en effet très petits. Les deux phases $A^{\prime}$ et $B^{\prime}$ partagent donc une très grande interface ce qui, du fait de leur immiscibilité induit une très grande énergie de surface. La minimisation de l'énergie du système passe alors par la diminution de l'interface entre les phases, donc par la croissance des domaines (fig. E2). En l'absence d'un contraste de densité significatif (facilitant la sédimentation) ou en cas de faible mobilité à l'échelle moléculaire, cette étape de mûrissement peut se révéler très lente. Selon les cas, la dynamique du mûrissement sera contrôlée par des mécanismes de diffusion ou de convection au sein du fluide [11].
Nucléation
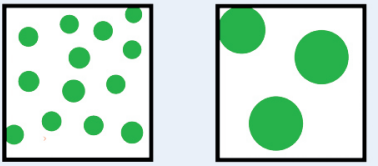

E2. Mûrissement.
Décomposition spinodale

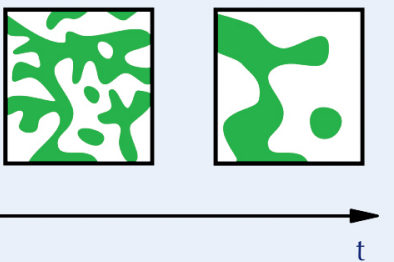

\section{Invariance d'échelle dynamique}

Le processus hors-équilibre de mûrissement est caractérisé par la croissance d'une longueur typique $/(T, t)$. À temps long, lorsque cette longueur est plus grande que toute échelle microscopique du système, s'établit une invariance d'échelle dynamique [12]. Ce type de symétrie est similaire à l'invariance d'échelle observée au voisinage d'un point critique à l'équilibre. La dynamique du système est alors entièrement contrôlée par la croissance de $/(\mathrm{T}, \mathrm{t})$, i.e. la dépendance en temps n'intervient que via la longueur caractéristique $l(T, t)$. Comme illustré sur la figure E3, avec la remise à l'échelle par un zoom de structures obtenues à des temps différents, cette invariance induit en particulier que la géométrie des domaines reste statistiquement identique à elle-même après renormalisation par $/(\mathrm{T}, \mathrm{t})$, et que l'évolution temporelle de $I(T, t)$ suit une loi de puissance avec le temps : $/(\mathrm{T}, \mathrm{t}) \approx \mathrm{t}^{\alpha}$.

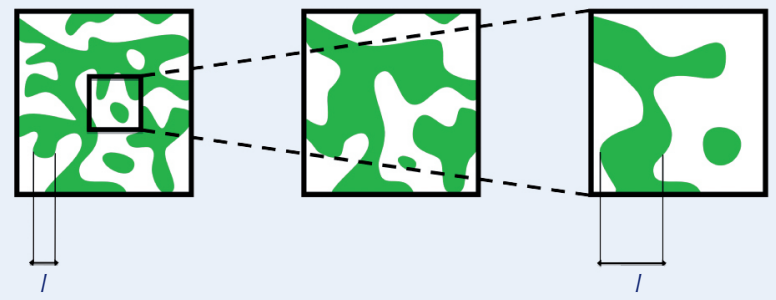

E3. Croissance et invariance d'échelle dynamique. 
remarque cependant que certaines des ruptures capillaires ont lieu au voisinage des extrémités libres de filaments, et forment ainsi des domaines isolés. La conséquence est très importante : dans le régime de croissance considéré, un domaine isolé ne peut plus contribuer au mûrissement ; la tension de surface en fait une goutte sphérique "piégée " dans cet état. Et, en effet, la fragmentation progressive de la phase la plus fluide ralentit le mûrissement visqueux et finalement, lorsque cette phase n'est plus composée que de gouttes isolées, l'arrête. D'autres mécanismes de mûrissement, plus lents (croissance diffusive, coalescence) peuvent alors prendre le relais à temps plus long.

La tomographie permet de caractériser finement ce processus de fragmentation, car chaque nouveau domaine peut être identifié individuellement.

Deux caractéristiques essentielles de cette fragmentation ont été mises en évidence.

D’une part, elle est intimement associée au mûrissement : les domaines isolés se détachent progressivement du domaine percolant, et comme la croissance continue chez ce dernier, au cours du temps les domaines qui se détachent sont de plus en plus gros. La conséquence de ce couplage est la formation d'une distribution de taille de gouttes (ou domaines isolés) en loi de puissance $\mathrm{p}(l) \sim l^{-5 / 3}$ jusqu'à une coupure $l_{c}$ qui est donnée par la fin de la percolation de la phase fragmentée.

D'autre part, on peut également comprendre l'asymétrie qui existe entre les deux phases. Les ruptures capillaires observées ici en tomographie sont en effet analogues aux phénomènes de fragmentation des filaments fluides. Ces derniers ont été amplement observés depuis Lord Rayleigh, au XIX ${ }^{\mathrm{e}}$ siècle, et on sait qu'un filament s'écoulant dans un liquide de viscosité plus faible est beaucoup plus stable qu'un filament fluide dans un liquide visqueux : le miel qui coule de la cuillère à la tartine ne forme pas de chapelet de gouttes comme l'eau coulant du robinet, et va même se rétracter plutôt que se rompre plus d'une fois. D'ailleurs, nos expériences montrent que les filaments de la phase moins visqueuse se rompent plus facilement, en particulier au niveau des extrémités libres, ce qui n'est pratiquement pas observé pour les filaments visqueux.
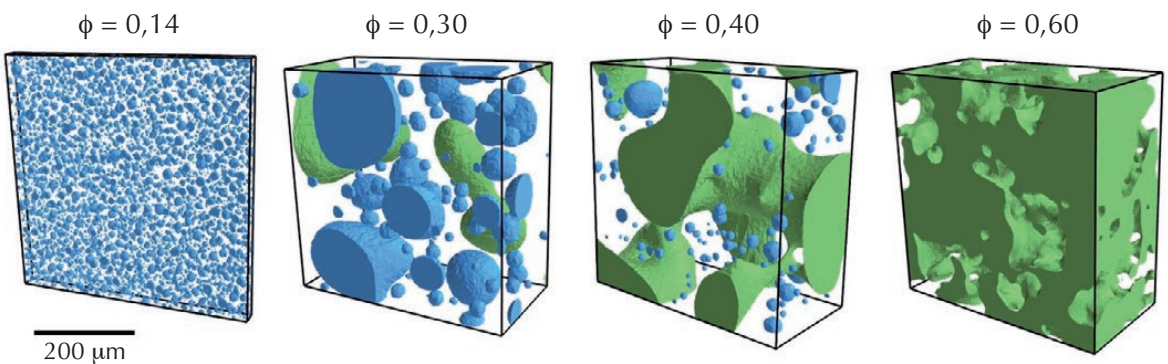

4. Morphologies obtenues aux temps longs en fonction de la fraction volumique $\phi$ de phase la moins visqueuse : de gauche à droite de $14 \%$ à $60 \%$. Seule la phase fluide est représentée. Les domaines isolés sont en bleu (et relaxent vers des sphères), le domaine interconnecté est en vert. Plus la fraction volumique de la phase fluide est faible, et plus sa fragmentation est rapide, ce qui détermine la plus grande taille de domaine atteignable lors du mûrissement.

\section{... et tout finit par s'arrêter!}

La mécanique des fluides des filaments étirés explique donc le comportement morphologique complexe de nos expériences. Lorsqu'on change progressivement de fraction volumique, d'une phase minoritaire visqueuse dans une matrice fluide à une phase minoritaire fluide dans une matrice visqueuse, on fait apparaitre le phénomène de fragmentation, qui s'amplifie au fur et à mesure que la fraction volumique change. Cette fragmentation va même déterminer l'arrêt de la croissance, qui survient donc d'autant plus vite que la phase peu visqueuse est minoritaire (fig. 4).

Outre la compréhension à l'échelle locale grâce à la mécanique des fluides, on peut tirer des observations tomographiques une conclusion assez paradoxale. La fragmentation progressive du domaine initialement percolant suit la loi d'échelle dynamique, tout en y mettant fin! En d'autres termes, tant qu'elle n'a pas sensiblement affecté le domaine percolant, on peut renormaliser toutes les grandeurs géométriques, y compris celles qui caractérisent les domaines fragmentés.

Au-delà de son intérêt fondamental, à l'interface entre hydrodynamique et physique statistique, l'étude par imagerie 3D du mûrissement des verres d'oxyde présente un intérêt technologique évident. Dans une perspective de nano- ou microtexturation des matériaux, elle permet d'envisager, au-delà de la maîtrise de la taille caractéristique, un contrôle de la morphologie des domaines via le passage d'une géométrie interconnectée à une géométrie de gouttelettes.

\section{Références}

1• D.R. Neuville, L. Cormier, D. Caurant et L. Montagne (éditeurs), Du verre au cristal, EDP Sciences (2013).

2 A.J. Bray, "Coarsening dynamics of phase-separating systems”, Phil. Trans. R. Soc. A 361 (2003) 781.

3• 0. Mazurin et E. Porai-Koshits (éditeurs), Phase Separation in Glass, North-Holland, Amsterdam (1984).

4 R.H. Doremus et A.M. Turkalo, "Phase separation in Pyrex glass", Science 164 (1969) 418.

$5 \bullet$ N. Ford et R. Todhunter, "Application of microporous glasses", dans M.H. Lewis, Glasses and Glass-ceramics, Springer (2013).

6• D. Bouttes, « Séparation de phase dans un verre borosilicate de baryum, du mûrissement à la fragmentation », Thèse, Université Pierre et Marie Curie (2014).

7•D. Bouttes et al., "Fragmentation and Limits to Dynamical Scaling in Viscous Coarsening: An Interrupted in situ X-Ray Tomographic Study", Phys. Rev. Lett., 112 (2014) 245701.

8• D. Bouttes et al., "Hydrodynamic coarsening in phase-separated melts“, Acta Materialia 92 (2015) 233.

9• E.D. Siggia, "Late stages of spinodal decomposition in binary mixtures", Phys. Rev. A 20, (1979) 595.

10• R.W. Balluffi, S.M. Allen et W. C. Carter, Kinetics of materials, Wiley (2005).

11• A. Onuki, Phase transitions dynamics, Cambridge University Press (2002).

12• L.F. Cugliandolo, "Out of equilibrium dynamics of complex systems", Notes de cours M2 (2015), www.Ipthe.jussieu.fr/ leticia/TEACHING/ cours-martine-march15.pdf . 